Int. J. Dev. Biol. 50: 481-489 (2006)

doi: $10.1387 / \mathrm{ijdb} .052067 \mathrm{js}$

Original Article

\title{
Proliferation and apoptosis in early molar morphogenesis - voles as models in odontogenesis
}

\author{
JANA SETKOVA*,1, HERVE LESOT'2,3, EVA MATALOVA ${ }^{1,5}$, KIRSTI WITTER $^{4}$, PETRA MATULOVA $^{1}$ \\ and IVAN MISEK ${ }^{1,5}$ \\ ${ }^{1}$ Laboratory of Animal Embryology, IAPG CAS CZ, Brno, Czech Republic ${ }^{2}$ UMR INSERM U595, Faculté de Medecine, Strasbourg, France \\ ${ }^{3}$ Université Louis Pasteur, Faculté de Chirurgie Dentaire, Strasbourg, France ${ }^{4}$ Department of Pathobiology, University of Veterinary \\ Medicine, Vienna, Austria and ${ }^{5}$ University of Veterinary and Pharmaceutical Sciences, Brno, Czech Republic
}

\begin{abstract}
Proliferation and apoptosis play crucial roles in the development of multicellular organisms. Their precise balance is necessary for tissue homeostasis throughout life. The developing dentition is a suitable model to study proliferation and apoptosis during embryogenesis, but the corresponding studies have been carried out principally in the mouse. The present study aimed to examine proliferation and apoptosis in the vole (Microtus sp., Rodentia) during the early morphogenesis of the first upper molar and compare it to what is known from the mouse. To this end, apoptosis and proliferation were investigated using histology and computer-aided 3D reconstruction. Mitoses accumulated predominantly in the developing cervical loop. Apoptosis during early odontogenesis showed highly specific spatio-temporal patterns in the dental epithelium. Apoptotic bodies were localised in non-dividing cell populations. They accumulated in the same places as described in the mouse: antemolar vestiges (ED 12.5 - 15.5), enamel knot (ED 14.5 - 15.5), stalk and palatally along the whole first molar tooth germ longitudinal axis (ED 15 15.5). Early tooth development in the field vole, including the distribution of apoptosis and mitosis, is very similar to that reported in the mouse, with the exception of the antemolar region. The microtine antemolar vestige is preserved longer than the murine one. It is conceivable that additional distinct differences in morphogenetic processes appear later in tooth development.
\end{abstract}

KEY WORDS: tooth development, morphogenesis, Microtus, dentition, 3D reconstruction

\section{Introduction}

Tooth development is a suitable model for studying morphogenetic events. Several studies have been focused on possible roles of cell proliferation and apoptosis during tooth morphogenesis (Lesot et al., 1996; Shigemura et al., 1999; Peterkova et al., 2003). Proliferation was investigated using complementary approaches all along tooth development (Casasco et al., 1992; Ruch, 1995; Lesot et al., 1996; Shigemura et al., 1999; Matulova et al., 2002). Mitosis in both dental epithelium and mesenchyme is involved in the development of the cervical loop in molars, together with the mesial elongation of the first molar (Lesot et al., 1996). During more advanced developmental stages, withdrawal from the cell cycle represents an initial step for odontoblasts and ameloblasts to engage in their functional differentiation (Casasco et al., 1992; Ruch, 1995).

Apoptosis occurs during all stages of tooth development, e.g. in early stages of morphogenesis (Lesot et al., 1996; Vaahtokari et al., 1996; Viriot et al., 1997; Jernvall et al., 1998; Lesot et al., 1999; Viriot et al., 2000, Matalova et al., 2004b), dentinogenesis
(Bronckers et al., 1996; Vermelin et al., 1996; Franquin et al., 1998), amelogenesis (Smith and Warshawsky, 1977; Moe, 1979; Nishikawa and Sasaki, 1995; Kaneko et al., 1997) and during eruption (Schellens et al., 1982; ten Cate and Anderson, 1986). The restricted distribution patterns of apoptotic cells at these different stages suggest multiple roles for apoptosis in dental development (Peterkova et al., 2003; Matalova et al., 2004a).

Although there is substantial evidence for essential roles of proliferation in embryogenesis and apoptosis in organ shaping and controlling of cell number (Meier et al., 2000; Doseff et al., 2004), only little is known in mammalian species other than the mouse and the rat. The vole and the mouse are closely related rodents with the same tooth formula (1.0.0.3/1.0.0.3). However, the shape of murine and microtine molars differs substantially. In the field vole, molars grow continuously, but not in the mouse. Moreover, the embryonic dental formulas seem to differ in both species (Witter et al., 1996; Keranen et al., 1999; Witter et al.,

Abbreviations used in this paper: 3D, three-dimensional; ED, embryonic day; EK, enamel knot; M1, first molar primordium.

*Address correspondence to: Dr. Jana Setkova. Laboratory of Animal Embryology, IAPG CAS CZ, Veveri 97, 602 00, Brno, Czech Republic.

Fax: +4205-4121-2988. e-mail: setkova@iach.cz 


\section{5) (Fig. 1).}

Differences of tooth morphology in both species could be caused by differences in proliferation and apoptosis during development. Proliferation and apoptosis during the odontogenesis of the field vole have been assessed by detection of the proliferation marker Proliferating Cell Nuclear Antigen (PCNA) (Matulova et al., 2002) and by TUNEL test (Matalova et al., 2004b), respectively. However, neither the spatial distribution of proliferating and apoptotic cells within the tooth primordia nor their relationship with tooth morphogenesis is known in detail.

This study aimed to show the three-dimensional (3D) distribution of proliferating and apoptotic cells within the first upper molar primordium (M1) of the vole and to compare these results with the situation in the mouse.

\section{Results}

In 3D reconstruction images of the maxillary molar region, three distinct structures were distinguishable: mesially the diastemal dental lamina, the antemolar vestige and distally M1 (Fig. 2). In all examined stages, distribution of mitoses was uniform in structures derived from the dental mesenchyme, but very rare in the antemolar region (not shown). Since apoptosis was very rare in the mesenchyme, only apoptosis in the epithelium was presented in serial drawings.

\section{ED 12 (82 $\mathrm{mg})$}

In the youngest animal under study, a distally decreasing dental lamina was observed in the mesial part of the maxillary cheek region (Fig. 2A). Few mitoses were uniformly distributed in both oral and dental epithelium. Apoptosis was almost absent at this stage of dental development (Fig. 3A).

\section{ED 12.5 (104 mg)}

In 3D reconstruction images (Fig. 2B), the diastemal dental lamina, the antemolar vestige and M1 could be easily distinguished, the antemolar vestige being the most prominent structure. The dental epithelium contained slightly more mitoses than the adjacent oral epithelium. Metaphases were distributed uniformly in both dental and oral epithelium. Apoptosis was found

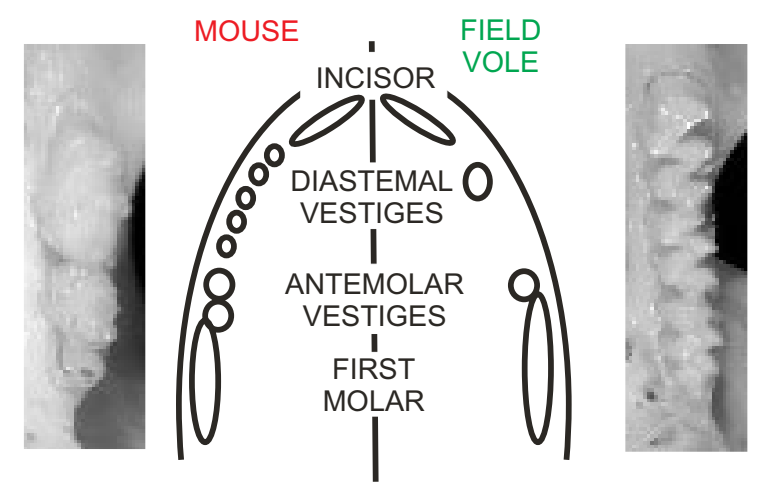

Fig. 1. Differences between the embryonic and adult upper dentition of the mouse and the field vole. The final tooth formula is the same in both species; however, the final molar tooth shape and the embryological tooth formula differ in both species. (Modified from Peterkova et al., 2003 and Witter et al., 2005.).
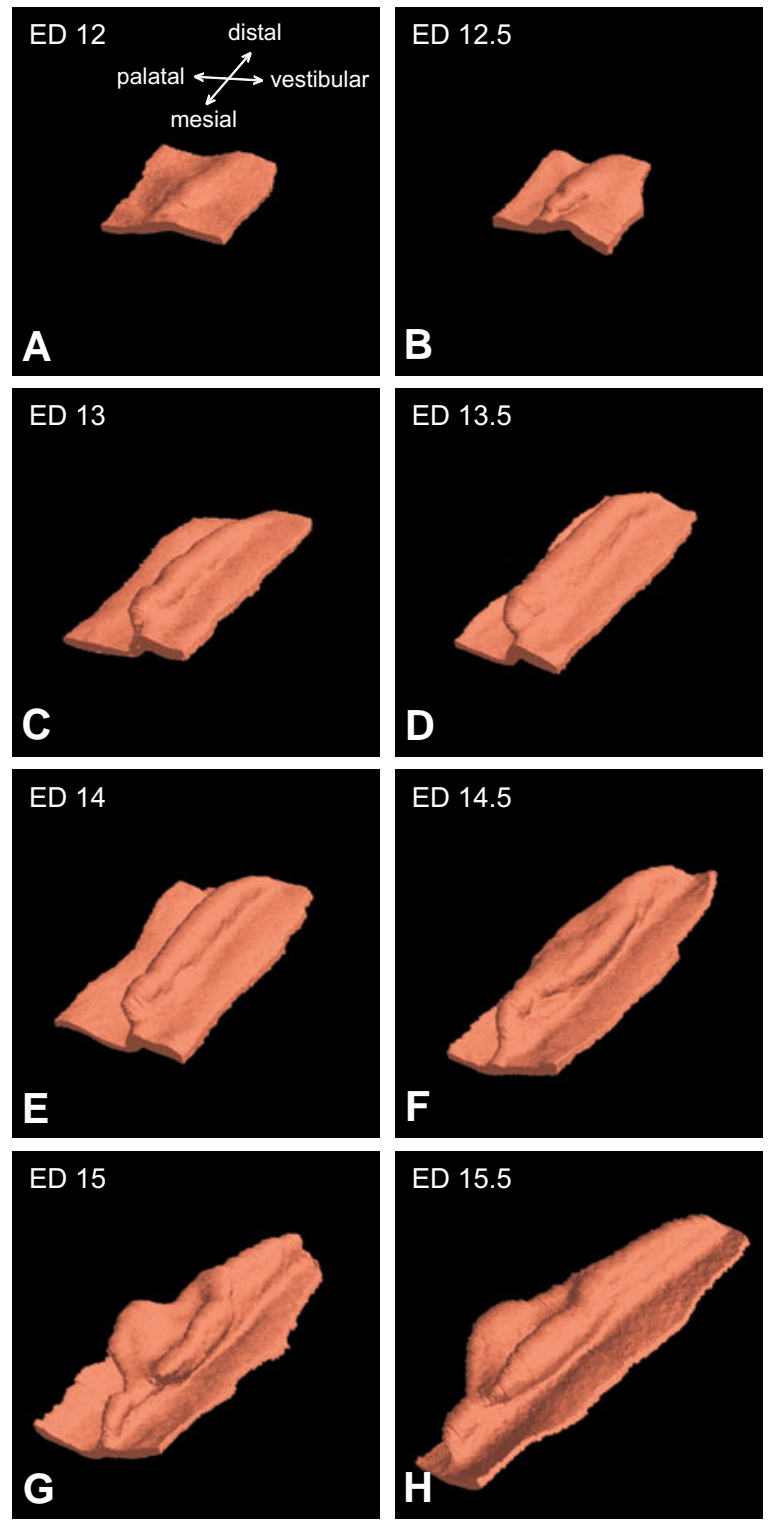

Fig. 2. Three dimensional reconstruction of the dental epithelium in the maxillary cheek region of the field vole (ED 12 - 15.5) showing the development of the first upper molar from dental lamina (ED 12), bud (ED 12.5 - 14), cap (ED 14.5 - 15), to early bell (ED 15.5). Mesio-palatal view.

almost only in the diastemal dental lamina and the antemolar vestige (Figs. 3B,4A).

\section{ED 13 (156 mg)}

At ED 13, the first molar tooth primordium increased and elongated in distal direction (Fig. 2C). The diastemal dental lamina started to regress. In frontal sections, the antemolar vestige had a shape of a prominent bud (Fig. 4B) that was followed by the smaller M1 bud. In the M1 epithelium, more mitoses could be found than in the adjacent oral epithelium (Fig. $3 \mathrm{C}$ ). Apoptosis was observed in the regressing diastemal dental lamina as well as in the antemolar vestige. In the antemolar vestige, apoptotic bodies accumulated at the tip of the epithelial bud forming a distinct cluster (Figs. 3C,4B). Despite of massive cell death, the 

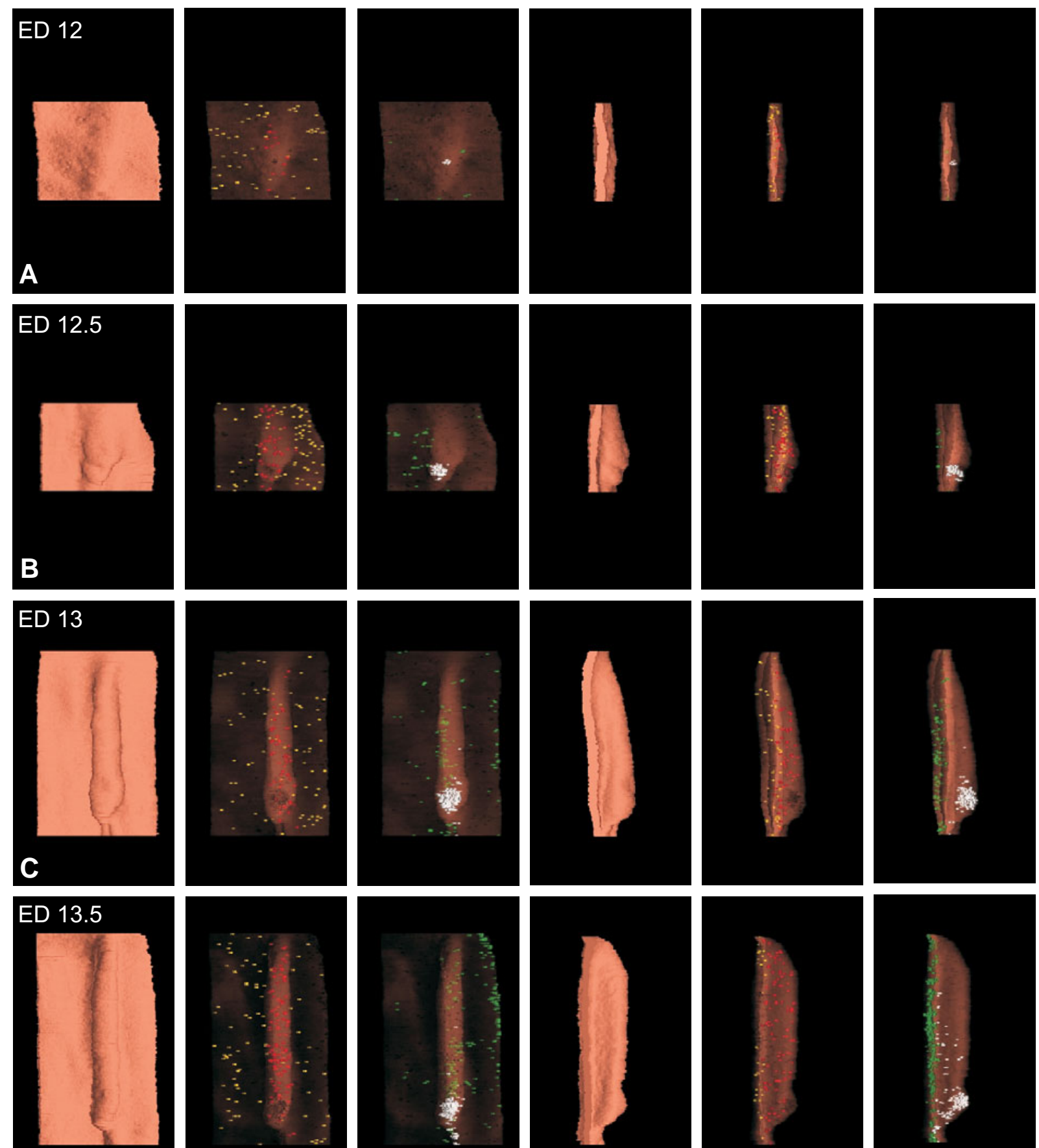

\section{D}
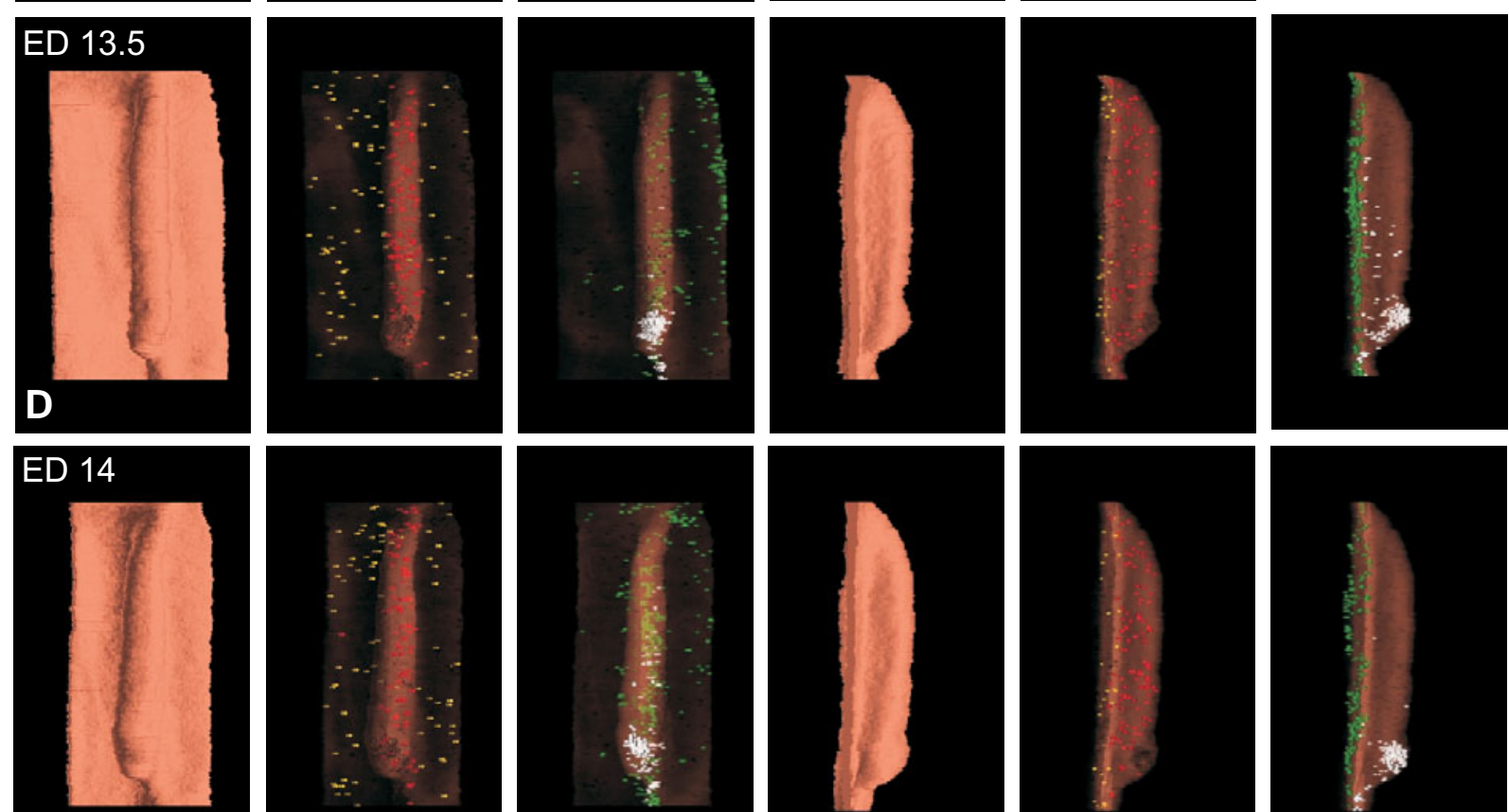

E
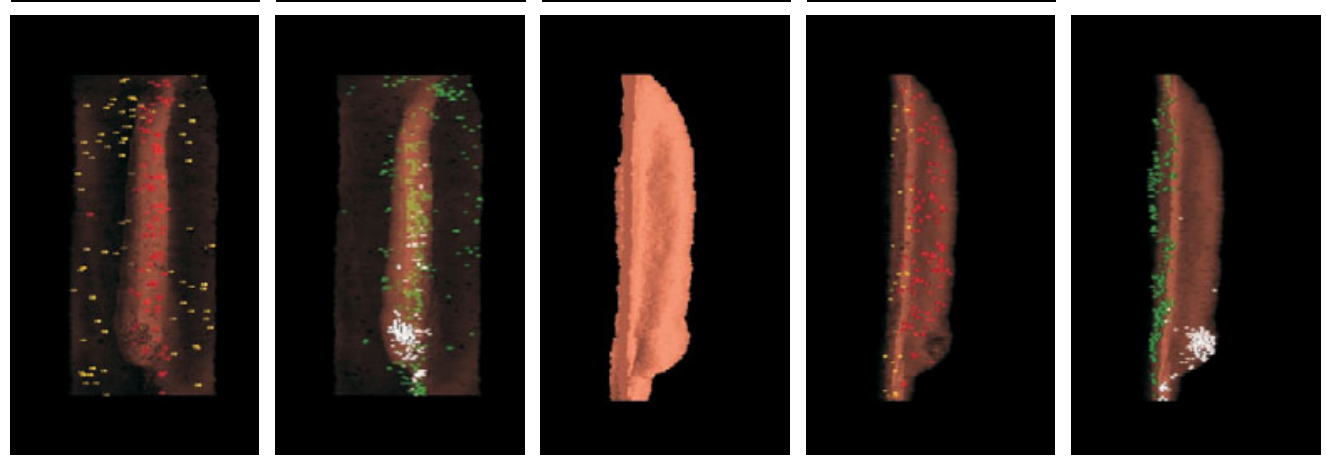

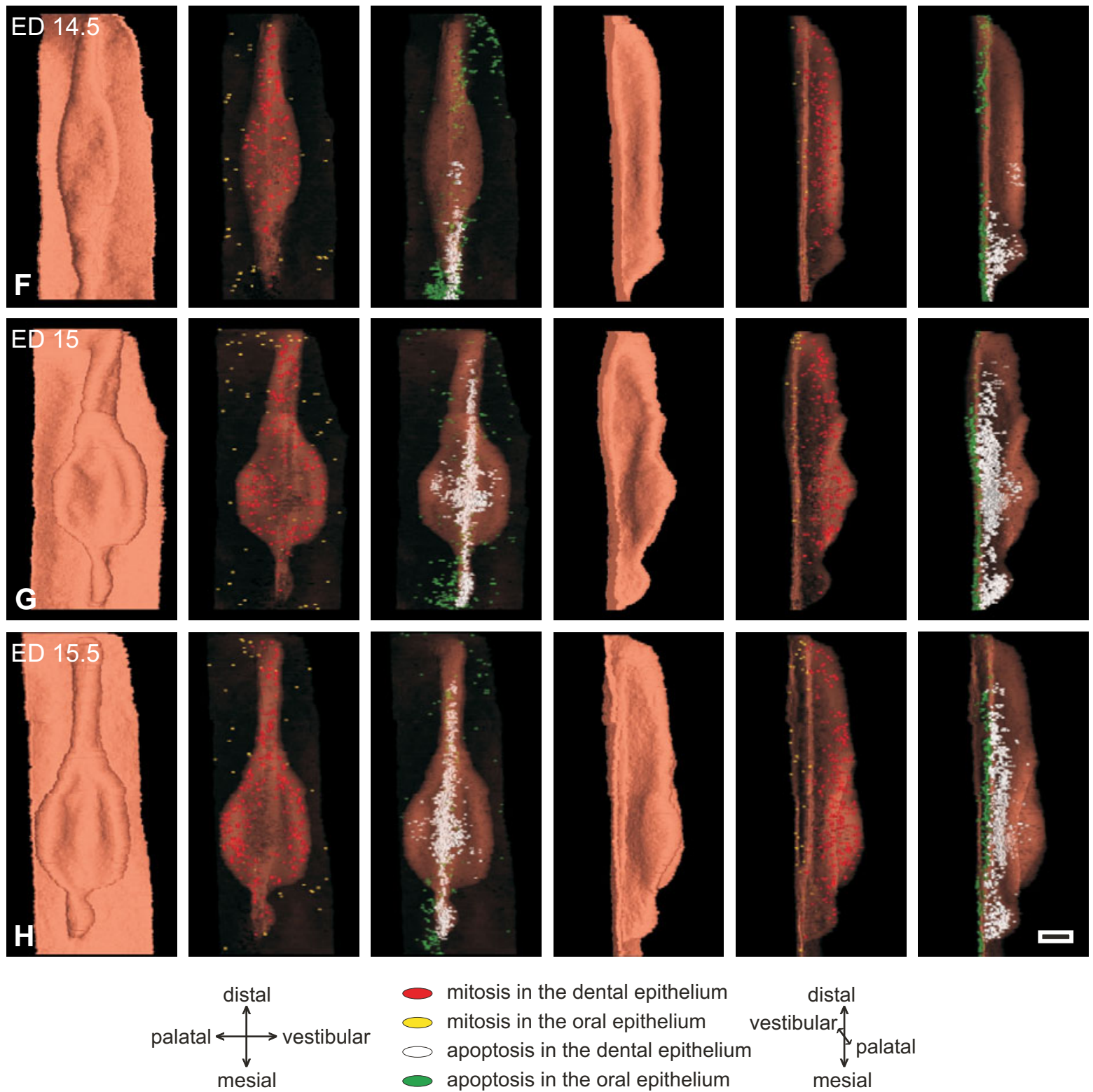
mitosis in the dental epithelium
$\checkmark$ mitosis in the oral epithelium
$\checkmark$ apoptosis in the dental epithelium
apoptosis in the oral epithelium

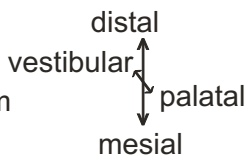

Fig. 3 (Previous page and above). Apoptosis and mitosis distribution in the first upper molar of the field vole. Three dimensional reconstruction (columns 1 and 4) and the distribution of metaphases (columns 2 and 5) and apoptotic bodies (columns 3 and 6) in the developing dental epithelium from ED 12 to ED 15.5. Red dots, dental mitosis; yellow dots, non-dental mitosis; white dots, apoptosis in dental epithelium; green dots, apoptosis in the non-dental epithelium. Bar represents $100 \mu \mathrm{m}$.

antemolar vestige was furthermore the most prominent part of the dental epithelium.

\section{ED 13.5 (177 mg)}

The regressing diastemal dental lamina was still apparent palatally from the developing vestibular lamina. The antemolar vestige developed into a large epithelial bud. A slight notch separated this structure from the long cylinder-like, distally narrowing M1 (Fig. 2D). In frontal sections, the antemolar vestige represented a narrow-based bud (Fig. 4C) whereas M1 was a wide-based tooth bud with surrounding concentrically condensed mesenchyme. Mitotic cells were distributed similarly to the previous developmental stage (Fig. 3D). Apoptotic cells clustered in the antemolar vestige. Additionally, there were few apoptotic bodies in the middle part of the M1. A third site of massive apoptosis was the connection of the stalk of the enamel organ with the oral epithelium (Figs. 3D,4C).

\section{ED $14(188 \mathrm{mg})$}

The dental epithelium had a similar shape as at the previous stage. However, the separation of the antemolar vestige from the M1 became less apparent (Fig. 2E). Mitotic cells were distributed as described at ED 13.5. Cell death was observed almost only in the diastemal dental lamina and the antemolar vestige. At sites of accumulation of apoptotic cells, no proliferation could be detected (Figs. 3E,4D). 


\section{ED 14.5 (275 mg)}

3 D reconstruction showed a regressing diastemal dental lamina, the antemolar vestige and an enlarged $\mathrm{M} 1$ with a flattened apical surface (early cap stage) (Fig. 2F). The antemolar vestige reached the late bud stage, its developmental maximum. ED 14.5 is the earliest stage when the primary enamel knot (primary EK) of M1 was distinguishable (Fig. 6A). It represented a slight ridge in the mesial part of M1. However, the primary EK protruded only slightly into the dental mesenchyme.

In contrast to the antemolar vestige with only few mitotic cells, M1 contained large proliferating cell populations, especially in the prospective cervical loop. In the primary EK region, no mitotic cells could be found (Figs. 3F,5A). Apoptotic bodies were detected in the primary EK as well as in the antemolar vestige. The characteristic accumulation of apoptotic bodies at the tip of the antemolar vestige disappeared at this stage, apoptotic bodies were dispersed in among the whole vestigial epithelial bud (Figs. $3 \mathrm{~F}, 4 \mathrm{E}, 5 \mathrm{~A}, 6 \mathrm{~A})$.

\section{ED 15 (339 mg)}

Antemolar vestige was still apparent, however it did not grow anymore. In the M1, the cervical loop started to elevate buccally and palatally around the mesenchyme of the prospective dental papilla. The papilla was surrounded by the epithelium laterally but neither mesially not distally (Fig. 2G). The epithelium of the tooth cap started to differentiate giving rise to inner and outer enamel epithelium as well as the stellate reticullum. In frontal sections, the primary EK had a round shape with concentrically arranged cells, however it did not protrude towards the dental papilla (Fig. 6B). Therefore, its overall shape could not be easily distinguished in $3 \mathrm{D}$ reconstruction images.

Mitoses were found particularly in the cervical loop enclosing the cap cavity. Interestingly, proliferating cells did not form clusters at the cervical loop edges, but were localised mainly in the outer enamel epithelium. Another cluster of mitotic cells was situated in the mesial part of the developing tooth cap. No mitosis was observed in the primary EK (Figs. 3G,5B).

Apoptosis was found in the antemolar vestige and in the primary EK. In the enamel knot, apoptotic cells were located in its deeper parts without contact to the basement membrane. A further cluster of apoptotic cells was observed also along the whole longitudinal axis of M1 between the palatal side of the enamel organ proper and its stalk forming a continuous band of apoptotic bodies, which connected the primary EK with the outer enamel epithelium on the palatal side of the tooth germ. However, this population of apoptotic bodies could not be assigned to any apparent morphological structure. In the M1 stalk, apoptosis concentrated predominantly in the most mesial and distal parts (Figs. 3G,4F,5B,6B).

\section{ED $15.5(385 \mathrm{mg})$}

The diastemal dental lamina already disappeared. The antemolar vestige was still apparent as a small bud mesially to the M1 early bell. Buccal and palatal cervical loop of M1 continued to elevate forming a deep cavity of the enamel organ, which opened mesially to the regressing antemolar vestige (Fig. $2 \mathrm{H}$ ). Primary EK was not as apparent as in the previous stage due to continuing regression (Fig. 6C).

Metaphases accumulated distinctly in the outer enamel epithe-
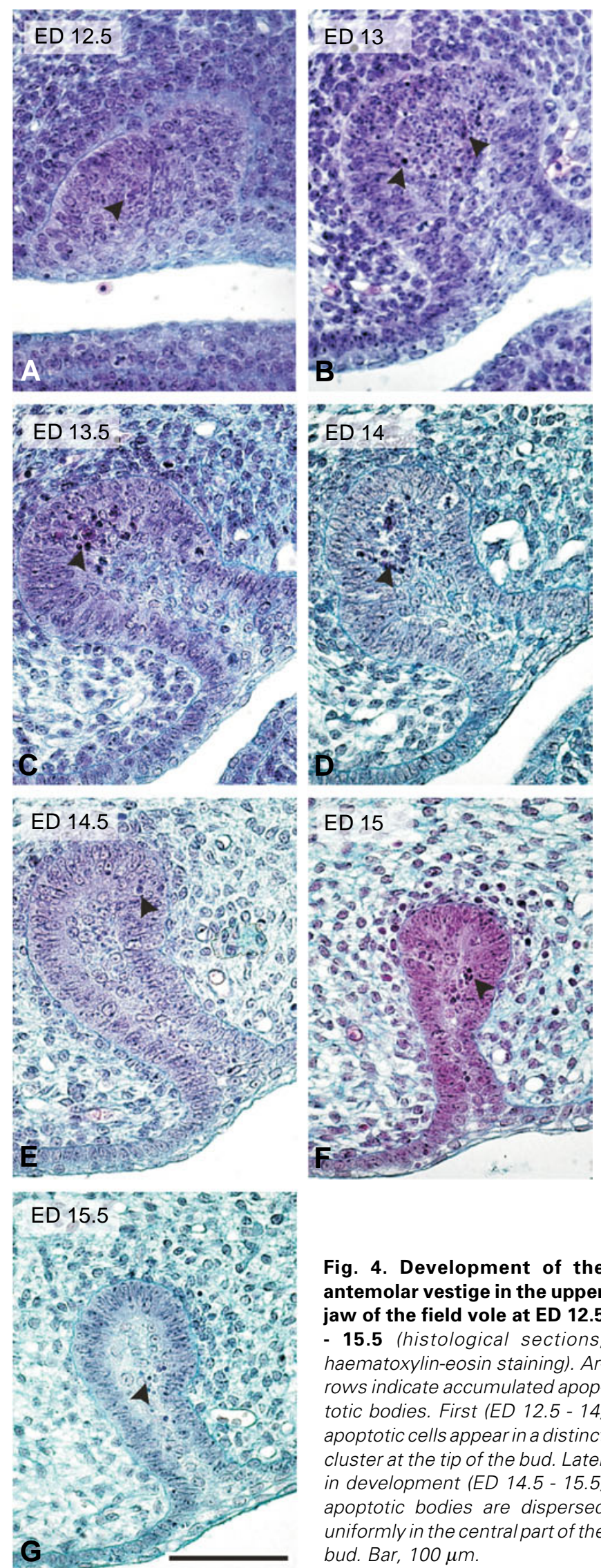

Fig. 4. Development of the antemolar vestige in the upper jaw of the field vole at ED $\mathbf{1 2 . 5}$ - 15.5 (histological sections, haematoxylin-eosin staining). Arrows indicate accumulated apoptotic bodies. First (ED 12.5 - 14) apoptotic cells appear in a distinct cluster at the tip of the bud. Later in development (ED 14.5 - 15.5) apoptotic bodies are dispersed uniformly in the central part of the bud. Bar, $100 \mu \mathrm{m}$. 

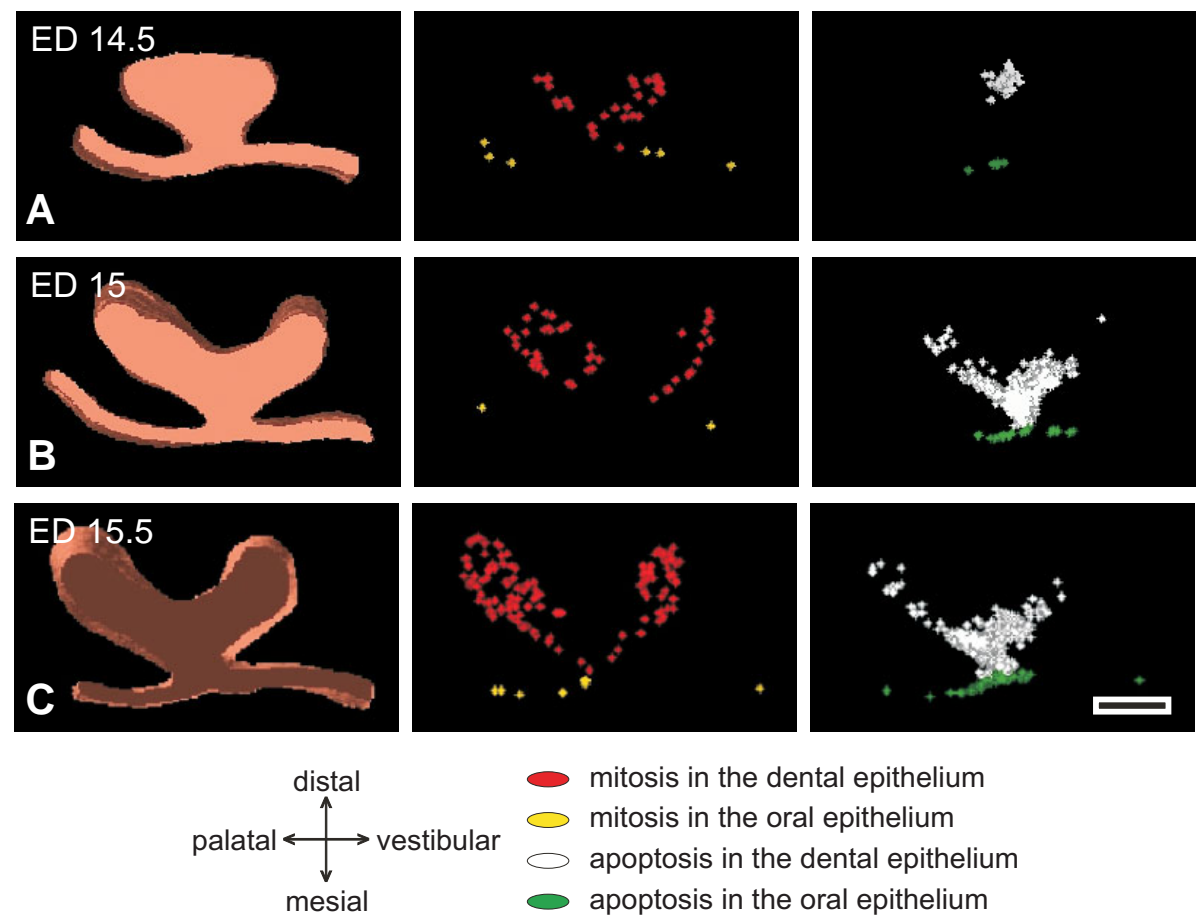

$\infty$ mitosis in the dental epithelium
mitosis in the oral epithelium
$\infty$ apoptosis in the dental epithelium
apoptosis in the oral epithelium

Fig. 5. Development of the primary enamel knot in the first upper molar primordium of the field vole at ED 14.5 - 15.5. Three-dimensional reconstruction of the enamel knot region of the tooth primordium (left column), distribution of mitotic (middle column) and apoptotic cells (right column) in this part of the dental epithelium. Red dots, dental mitosis; yellow dots, non-dental mitosis; white dots, dental apoptosis; green dots, non-dental apoptosis. Mesio-distal view. Bar represents $100 \mu \mathrm{m}$.

lium of both buccal and palatal cervical loop (Figs. 3H,5C).

Apoptotic cells were localised predominantly between primary EK and currently evolving stellate reticullum. Additionally, apoptotic cells concentrated along the whole tooth germ axis as described in the pervious stage, in the stalk of the enamel organ and the antemolar vestige (Figs. $3 \mathrm{H}, 4 \mathrm{G}, 5 \mathrm{C}, 6 \mathrm{C}$ ).

\section{Discussion}

Voles are an interesting model for comparative and developmental odontogenetic studies. The mouse and the vole dentitions are very similar but not the same. Different molar cusp patterns in both animal species could help to study the regulation of different tooth shape formation. Not only morphology, but also prenatal tooth development differs between the vole and the mouse. Differences in the development of molar shape are even more striking, particularly the formation of the mesial end of the first upper molar (Witter et al., 1996; Witter et al., 2005). In the mouse upper jaw, two antemolar vestiges (R1 and R2) regress by apoptosis and are 'overrolled' by the mesially elongating M1 (Peterkova et al., 2002). In the field vole, only one antemolar vestige has been found corresponding to the $\mathrm{R} 1$ element in the mouse. The first molar of the vole could therefore be made of 'more' dental epithelium than the compact first molar of the mouse (Witter et al., 2005).

Proliferation and apoptosis can be expected to take part in shaping of molar primordia. In the field vole, these processes were studied using immunohistochemical detection of PCNA and
TUNEL method, respectively (Matalova $e t$ al., 2004b; Matulova et al., 2002). PCNA was detected in cycling cells in both dental epithelium and mesenchyme, but not in antemolar vestiges, enamel knots and differentiated ameloblasts and odontoblasts (Matulova et al., 2002). Apoptosis was observed in PCNA negative areas in the middle axis of the tooth bud and later in the enamel knot region and in the stalk. Moreover, proapoptotic molecules (FasR, FasL) and caspase 3 activation have been documented in apoptosis areas of the field vole first upper molar tooth germ (Matalova et al., 2005; Matalova et al., 2006). However, a 3D study of proliferation and apoptosis in the field vole was still missing and this study aimed to tackle this problem. Since developmental changes in morphogenetic processes determining the future cusp patterning in mouse and vole lower molars occur before the first cusp is morphologically apparent (Jernvall et al., 2000), early molar morphogenesis (ED 12 - 15.5) of the field vole was investigated in our study to follow spatio-temporal distribution of apoptosis and mitosis in the epithelial and mesenchymal compartment.

As in the mouse (Lesot et al., 1996; Peterkova et al., 2000; Peterkova et al., 2002), early morphogenesis of the first upper molar primordium in the vole includes (i) apoptotical elimination or involvement of antemolar vestiges in development of the tooth primordium, (ii) elevation of the cervical loop and formation of the mesial border of the tooth cap and (iii) development and regression of the primary enamel knot. 3D distribution of mitosis and apoptosis indicates involvement of proliferation and/or apoptosis in all these processes.

Proliferation was investigated as metaphases distribution. Metaphases in the upper field vole molar region showed a rather uniform distribution although they accumulated first at ED 14.5 around the primary EK and later in the developing cervical loop (ED 14.5, 15, 15.5) predominantly in its deeper part, causing the cervical loop growth. At ED 15 metaphases accumulated in the anterior part of the tooth cap probably causing the elevation of the anterior part of the cervical loop and mesial elongation of the tooth primordium. Elevation of palatal and vestibular cervical loop in the vole seemed to result from cell proliferation, as described in the mouse (Lesot et al., 1996).

No metaphases, as expected, (ED 14.5 - 15.5), were found in the primary enamel knot area at the cap stage. Enamel knots are signalling centres suggested to control tooth crown morphogenesis. The primary enamel knot is characterised as an area of non-dividing cells in the centre of the tooth bud to bell and is believed to be essential for the transition from the bud to cap stage. They express a wide range of regulatory genes including Shh, Fgf, Wht and Bmp family members (for overview, see http://bite-it.helsinki.fi/ 2003), connected to a complex regulatory network that may determine the position of secondary enamel knots and further the localisation of cusps in the prospective tooth 
(Laurikkala et al., 2003).

Apoptosis is understood as a general mechanism whereby the enamel knot structure is eliminated. Apoptotic bodies were found in the primary EK of the vole, predominantly in its inner part - progressively shifting deeper into the enamel organ. No apoptosis was observed in the cell layer adjacent to the basal lamina. Additionally, apoptosis was extended toward the palatal part of the outer enamel epithelium, forming a continuous zone connecting the primary EK with the outer enamel epithelium. Apoptosis was located along the whole longitudinal tooth germ axis palatally at the border between the enamel organ and stalk, where expression of proapoptotic molecules (FasR and FasL) has been previously shown (Matalova et al., 2005). In this region, apoptosis was also found in the mouse (Lesot $e t$ al., 1996). However, compared to the field vole it is not as massive and not concentrated in such a well-characterised cluster. In the sibling vole, apoptosis at the border
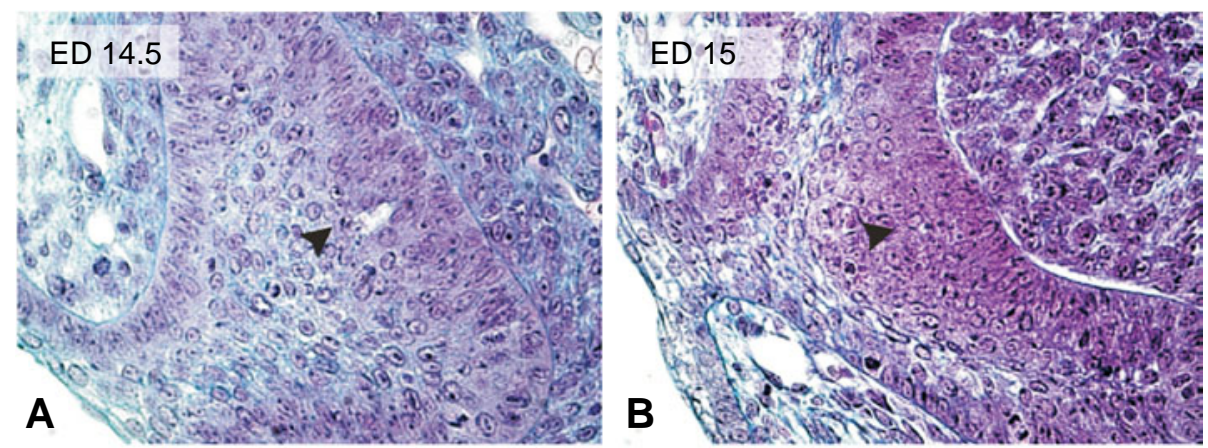

Fig. 6. Development of the primary enamel knot in the first upper molar of the field vole at ED 14.5 - $\mathbf{1 5 . 5}$ (histological sections, haematoxylin-eosin staining). Arrows indicate the primary enamel knot structure with accumulated apoptotic bodies. The primary enamel knot is a cluster of concentrically arranged nondividing cells that seems to be the signalling centre regulating transition from the bud to cap stage. This structure is terminated by apoptosis. Bar represents $100 \mu \mathrm{m}$.

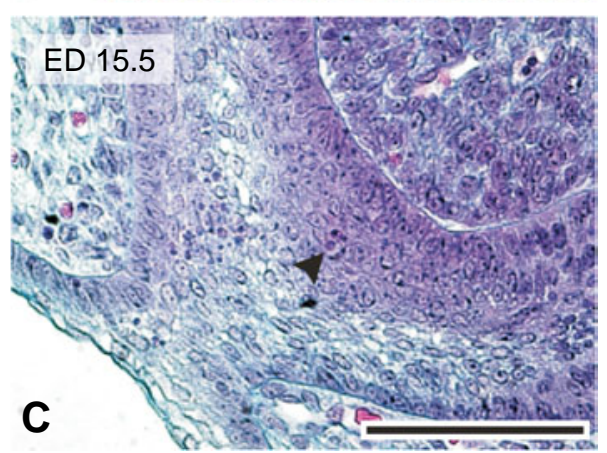

between enamel organ and its stalk has not been described. This cluster of apoptotic cells has not been assigned to any specific structure and its function is not yet clear. In the mouse and the sibling vole, no data about of any regulatory molecules specifically expressed in this area have been published yet.

Regression of antemolar vestiges is probably the most important morphogenetic process in the early molar morphogenesis in both the field vole and the mouse. Compared to the mouse with two antemolar vestiges and thus two clusters of apoptotic cells in the antemolar region (Peterkova et al., 2000), there is only one antemolar vestige with one corresponding cluster of apoptotic cells in the field vole, as confirmed by 3D reconstruction (Fig. 3).

Massive apoptosis was found in the antemolar region in the field vole upper molar from ED 12.5 toward ED 15.5. The distribution pattern of apoptotic bodies changed between ED 14 and ED 14.5. Until ED 14.5, a clearly defined area of apoptotic cells was observed, whereas, apoptotic bodies were scattered in the whole antemolar vestige at later stages.

Any similar vestigial structure has not been reported in the sibling vole. However, an epithelial signalling centre has been described in the first upper molar at ED 12 (Keranen et al., 1999), at the stage when the antemolar vestige can be easily mistaken with the M1. The early epithelial signalling centre in the sibling vole might be located in a structure homologous to the antemolar vestige in the field vole and one or both antemolar vestiges in the mouse. This hypothesis is supported by our findings of a specific

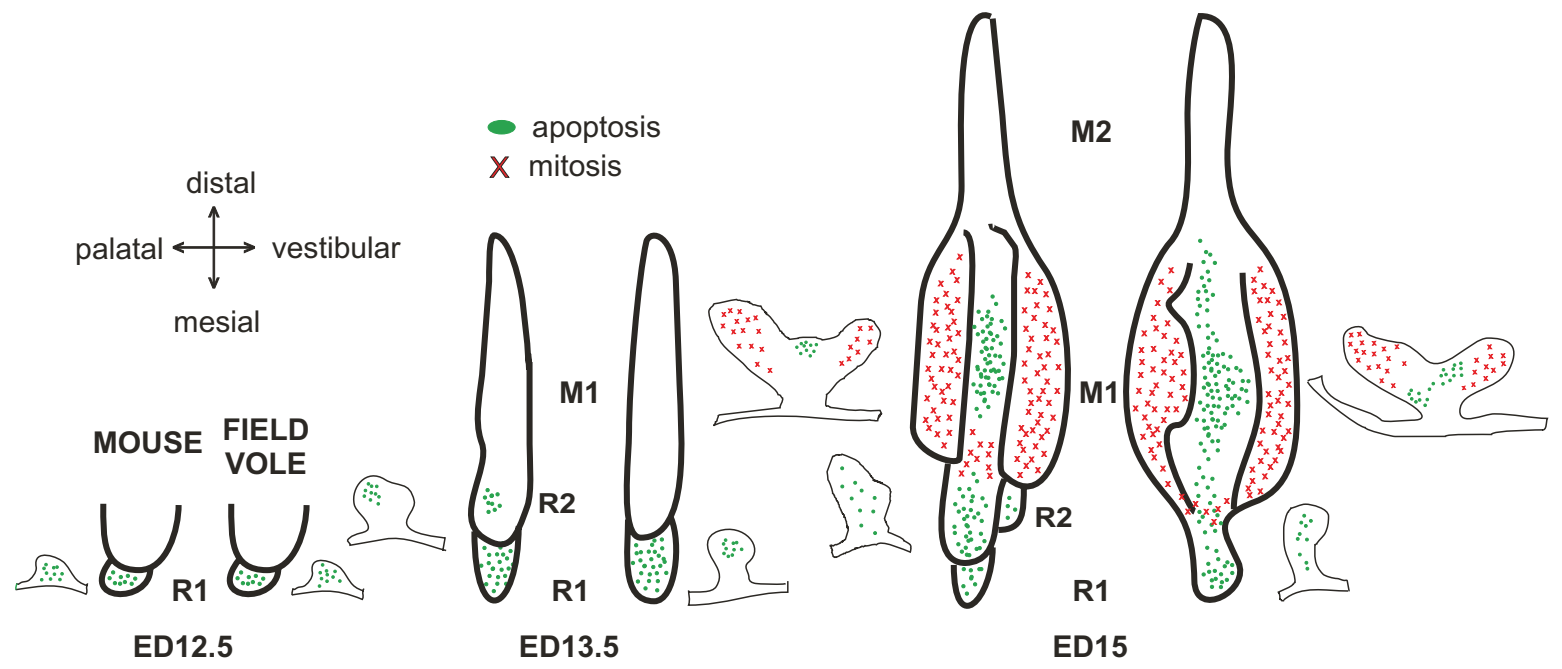

Fig. 7. Schematic drawings of the distribution of mitosis and apoptosis in the mouse and the field vole first upper molar primordium at ED 12.5 - 15. No major differences between first upper molar early morphogenesis in the mouse and field vole were found. However, two antemolar vestiges regress in the mouse upper molar region $(R 1, R 2)$, whereas in the field vole only one antemolar vestige was found. (Mouse schematic drawings are modified from Lesot et al., 1996; Peterkova et al., 2000). 
cluster of apoptotic cells at the tip of the field vole antemolar vestige between ED 12.5 and ED 14, which could correspond to the apoptotic removal of already silenced early epithelial signalling centre cells. Thus, the first period of apoptosis in the field vole antemolar vestige may correspond most likely to the apoptotic elimination of silenced early epithelial signalling centre cells, whereas, the second period of apoptosis causes its regression. Complete regression of the field vole antemolar vestige is reported after ED 17 (Witter et al., 2005). For the future, verification of candidate molecules involved in regression of the antemolar vestiges in the field vole is very challenging. The role of Msx-2, Bmp-2, Bmp-4, Fgf- 8 and Pax-9 proteins whose down-regulation seems to mediate the regression of vestigial buds in the mouse and sibling vole (Tureckova et al., 1995; Peterkova et al., 1998; Keranen et al., 1999; Yamamoto et al., 2005) should be of particular interest.

The molecular basis of evolutionary differences between the microtine and murine dentitions has been investigated in the sibling vole (Keranen et al., 1998; 1999; Jernvall et al., 2000; Salazar-Ciudad and Jernvall, 2002). The same genes (Bmp-2, Bmp-4, Fgf-4, Shh, Lef-1, Msx-1, Msx-2, p21) were expressed in the developing microtine and mouse lower molars, but in slightly different positions and timing. Thus, the same molecular cascades underlie the general cusp patterns in both microtine and murine molars and changes in tooth shapes probably result from slight spatio-temporal variations in gene expression (Keranen et al., 1998), which cause - by yet little known ways - similarly slight shifts in maximal proliferation activity and apoptosis, as observed in this study (Fig.7).

Voles are suitable models to test hypotheses proposed from studying odontogenesis in the mouse and to search for evolutionary differences between different animal species at cellular and molecular levels. For microtine and murine molars, a model predicting different tooth shapes was developed, showing general developmental processes participating in evolutionary tooth shape alterations (Salazar-Ciudad and Jernvall, 2002). According to our findings, no major differences in apoptosis and mitosis distribution during early tooth development can be observed between the mouse and the field vole. However, also minor changes reported in this article (Fig. 7) could give rise to more complex morphological differences further in development and finally in the tooth shape. Additionally, further functional experiments should elucidate, if apoptosis and proliferation play active morphogenetic roles and how far their interplay is necessary to achieve the proper tooth shape, size, formation and position in the jaws.

\section{Materials and Methods}

\section{Animals, specimens and histology}

Field vole embryos for this study were obtained from the embryological collection of the Institute of Animal Physiology and Genetics of the Academy of Sciences of the Czech Republic in Brno (http:// www.iapg.cas.cz/uzfg/lge/sbirkae.htm, 2003). Embryos were harvested and fixed in the Bouin's solution, processed and embedded in paraffin.

According to their age and weight (total weight without foetal membranes prior to fixation) (Peterka et al., 2002), heads of eight embryos corresponding to ED 12, 12.5, 13, 13.5, 14, 14.5, 15 and 15.5 were chosen. $5 \mu \mathrm{m}$ frontal serial sections were prepared and stained with haematoxylin-eosin.

\section{Mitosis and apoptosis evaluation}

Metaphases and apoptotic bodies were detected by morphological criteria. The location of proliferating cell populations within the developing teeth of the vole has been previously confirmed by immunohistochemical detection of proliferation marker PCNA (Matulova et al., 2002). The apoptotic nature of the condensed nuclear bodies has been previously confirmed by TUNEL staining (Matalova et al., 2004b).

\section{$3 D$ reconstruction}

Serial drawings of the left maxillary dental and adjacent oral epithelium were made at a magnification 400x using a Leica DMLB microscope equipped with a drawing chamber (Leica Microsystems, Vienna, Austria). Apoptotic bodies and mitotic cells (metaphases only) were marked in the drawings. The best-fit procedure (Gaunt and Gaunt, 1978) was used for superposition of the serial drawings. These were digitised and correlated using a Hamamatsu C2400 camera connected to a digital imaging system (Lesot et al., 1996). Software used for image acquisition and treatment was specially developed and adapted for this work. 3D images were generated by a commercial volume-rendering program (Sun Voxel, Sun Microsystems).

\section{Acknowledgement}

We wish to thank Mrs Zdenka Matousova and Ms Gabriela Prazanova for their excellent technical support. This study was supported by the Grant Agency of the Czech Republic (304/04/0101) and COST B 23 Programme (grant OC B23.001 and COST STSM B23-00981). Research in Brno lab runs in the frame of IRP IAPG No. AVOZ 50450515.

\section{References}

BRONCKERS A.L., LYARUU D.M., GOEI W., LITZ M., LUO G., KARSENTY G., WOLTGENS J.H. and D'SOUZA R.N. (1996). Nuclear DNA fragmentation during postnatal tooth development of mouse and hamster and during dentin repair in the rat. Eur. J. Oral Sci. 104:102-111.

CASASCO A., CALLIGARO A. and CASASCO M. (1992). Proliferative and functional stages of rat ameloblast differentiation as revealed by combined immunocytochemistry against enamel matrix proteins and bromodeoxyuridine. Cell Tissue Res. 270:415-423.

CATE A.R. and ANDERSON R.D. (1986). An ultrastructural study of tooth resorption in the kitten. J. Dent. Res. 65:1087-1093.

DOSEFF A.I. (2004). Apoptosis: the sculptor of development. Stem Cells Dev. 13:473-483.

FRANQUIN J.C., REMUSAT M., ABOU HASHIEH I. and DEJOU J. (1998). Immunocytochemical detection of apoptosis in human odontoblasts. Eur. J. Oral Sci. 106:384-387.

GAUNT P.N. and GAUNT W.A. (1978). Three dimensional reconstruction in biology. Pitman medical publishing co. Tunbridge Wells.

JERNVALL J., ABERG T., KETTUNEN P., KERANEN S. and THESLEFF I. (1998). The life history of an embryonic signaling center: BMP-4 induces p21 and is associated with apoptosis in the mouse tooth enamel knot. Development. 125:161-169.

JERNVALL J., KERANEN S.V. and THESLEFF I. (2000). Evolutionary modification of development in mammalian teeth: quantifying gene expression patterns and topography. Proc. Natl. Acad. Sci. USA. 97:14444-14448.

KANEKO H., OGIUCHI H. and SHIMONO M. (1997). Cell death during tooth eruption in the rat: surrounding tissues of the crown. Anat. Embryol. (Berl). 195:427-434.

KERANEN S.V., ABERG T., KETTUNEN P., THESLEFF I. and JERNVALL J. (1998). Association of developmental regulatory genes with the development of different molar tooth shapes in two species of rodents. Dev. Genes Evol. 208:477-486.

KERANEN S.V., KETTUNEN P., ABERG T., THESLEFF I. and JERNVALL J. (1999). Gene expression patterns associated with suppression of odontogenesis in mouse and vole diastema regions. Dev. Genes Evol. 209:495-506.

LAURIKKALA J., KASSAIY., PAKKASJARVI L., THESLEFF I. and ITOH N. (2003). 
Identification of a secreted BMP antagonist, ectodin, integrating BMP, FGF and SHH signals from the tooth enamel knot. Dev. Biol. 264:91-105.

LESOT H., PETERKOVA R., SCHMITT R., MEYER J.M., VIRIOT L., VONESCH J.L., SENGER B., PETERKA M. and RUCH J.V. (1999). Initial features of the inner dental epithelium histo-morphogenesis in the first lower molar in mouse. Int. J. Dev. Biol. 43:245-254.

LESOT H., VONESCH J.L., PETERKA M., TURECKOVA J., PETERKOVA R. and RUCH J.V. (1996). Mouse molar morphogenesis revisited by three-dimensional reconstruction. II. Spatial distribution of mitoses and apoptosis in cap to bell staged first and second upper molar teeth. Int. J. Dev. Biol. 40:1017-1031.

MATALOVA E., KOVARU F. and MISEK I. (2006). Caspase 3 activation in the primary enamel knot of developing molar tooth. Physiol. Res. 55: (in press).

MATALOVA E., TUCKER A.S. and MISEK I. (2005). Apoptosis-related factors (Fas receptor, Fas ligand, FADD) in early tooth development of the field vole (Microtus agrestis). Arch. Oral Biol. 50:165-169.

MATALOVA E., TUCKER A.S. and SHARPE P.T. (2004a). Death in the life of a tooth. J. Dent. Res. 83:11-16.

MATALOVA E., WITTER K. and MISEK I. (2004b). Apoptosis distribution in the first molar tooth germ of the field vole (Microtus agrestis). Tissue Cell. 36:361-367.

MATULOVA P., WITTER K. and MISEK I. (2002). Proliferating cell nuclear antigen (PCNA) expression in tooth primordia in the field vole (Microtus agrestis, Rodentia). Connect. Tissue Res. 43:138-142.

MEIER P., FINCH A. and EVAN G. (2000). Apoptosis in development. Nature. 407:796-801.

MOE H. (1979). Physiological cell death of secretory ameloblasts in the rat incisor. Cell Tissue Res. 197:443-451.

NISHIKAWA S. and SASAKI F. (1995). DNA localization in nuclear fragments of apoptotic ameloblasts using anti-DNA immunoelectron microscopy: programmed cell death of ameloblasts. Histochem. Cell Biol. 104:151-159.

PETERKA M., LESOT H. and PETERKOVA R. (2002). Body weight in mouse embryos specifies staging of tooth development. Connect. Tissue Res. 43:186190.

PETERKOVA R., PETERKA M. and LESOT H. (2003). The developing mouse dentition: a new tool for apoptosis study. Ann. N Y Acad. Sci. 1010:453-466.

PETERKOVA R., PETERKA M., VIRIOT L. AND LESOT H. (2000). Dentition development and budding morphogenesis. J. Craniofac. Genet. Dev. Biol. 20:158-172.

PETERKOVA R., PETERKA M., VIRIOT L. and LESOT H. (2002). Development of the vestigial tooth primordia as part of mouse odontogenesis. Connect. Tissue Res. 43:120-128.

PETERKOVA R., PETERKA M., VONESCH J.L., TURECKOVA J., VIRIOT L., RUCH J.V. and LESOT H. (1998). Correlation between apoptosis distribution and BMP-2 and BMP-4 expression in vestigial tooth primordia in mice. Eur. J. Oral Sci. 106:667-670.

RUCH J.V. (1995). Tooth crown morphogenesis and cytodifferentiations: candid questions and critical comments. Connect. Tissue Res. 32:1-8.
SALAZAR-CIUDAD I. and JERNVALL J. (2002). A gene network model accounting for development and evolution of mammalian teeth. Proc. Natl. Acad. Sci. USA. 99:8116-8120.

SCHELLENS J.P., EVERTS V. and BEERTSEN W. (1982). Quantitative analysis of connective tissue resorption in the supra-alveolar region of the mouse incisor ligament. J. Periodontal Res. 17:407-422.

SHIGEMURA N., KIYOSHIMA T., KOBAYASHI I., MATSUO K., YAMAZA H., AKAMINE A. and SAKAI H. (1999). The distribution of BrdU- and TUNELpositive cells during odontogenesis in mouse lower first molars. Histochem. J. 31:367-377.

SMITH C.E. and WARSHAWSKY H. (1977). Quantitative analysis of cell turnover in the enamel organ of the rat incisor. Evidence for ameloblast death immediately after enamel matrix secretion. Anat. Rec. 187:63-98.

TURECKOVA J., SAHLBERG C., ABERG T., RUCH J.V., THESLEFF I. and PETERKOVA R. (1995). Comparison of expression of the msx-1, msx-2, BMP2 and BMP-4 genes in the mouse upper diastemal and molar tooth primordia. Int. J. Dev. Biol. 39:459-468.

VAAHTOKARI A., ABERG T. and THESLEFF I. (1996). Apoptosis in the developing tooth: association with an embryonic signaling center and suppression by EGF and FGF-4. Development. 122:121-129.

VERMELIN L., LECOLLE S., SEPTIER D., LASFARGUES J.J. and GOLDBERG M (1996). Apoptosis in human and rat dental pulp. Eur. J. Oral Sci. 104:547-553.

VIRIOT L., LESOT H., VONESCH J.L., RUCH J.V., PETERKA M. and PETERKOVA R. (2000). The presence of vestigial odontogenic structures in the mouse embryonic mandible requires reinterpretation of developmental control of first lower molar histomorphogenesis. Int. J. Dev. Biol. 44:233-240.

VIRIOT L., PETERKOVA R., VONESCH J.L., PETERKA M., RUCH J.V. and LESOT H. (1997). Mouse molar morphogenesis revisited by three-dimensional reconstruction. III. Spatial distribution of mitoses and apoptoses up to bellstaged first lower molar teeth. Int. J. Dev. Biol. 41:679-690.

WITTER K., LESOT H., PETERKA M., VONESCH J.L., MISEK I. and PETERKOVA R. (2005). Origin and developmental fate of vestigial tooth primordia in the upper diastema of the field vole (Microtus agrestis, Rodentia). Arch. Oral Biol. 50:401409.

WITTER K., MISEK I., PETERKA M. and PETERKOVA R. (1996). Stages of odontogenesis in the field vole (Microtus agrestis, Rodentia) - A pilot study. Acta vet. Brno. 65:285-296.

YAMAMOTO H., CHO S.W., SONG S.J., HWANG H.J., LEE M.J., KIM J.Y. and JUNG H.S. (2004). Characteristic tissue interaction of the diastema region in mice. Arch. Oral Biol. 50:189-198.

http://bite-it.helsinki.fi/ (2003).

http://www.iapg.cas.cz/uzfg/lge/sbirkae.htm (2003).

Received: August 2005

Reviewed by Referees: October 2005

Modified by Authors and Accepted for Publication: December 2005 